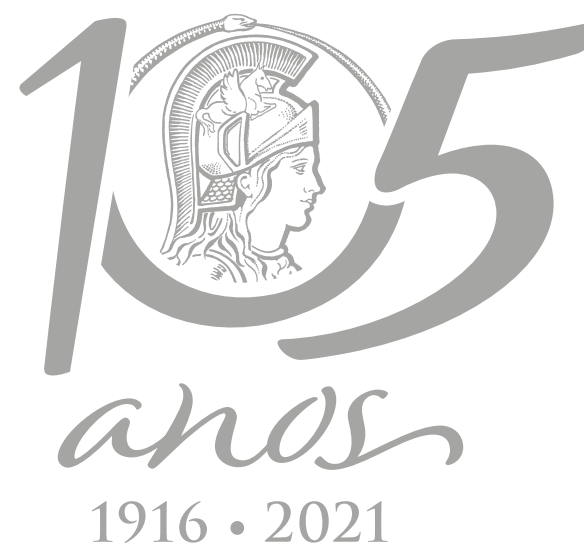

\title{
Astragalus polysaccharide attenuates overexercise-induce myocardial injury via activating AMPK signaling pathway to suppress inflammation and oxidative stress
}

\author{
XINLING TUO, ZHIJIAN DENG, GUOCHAO HUANG, HUIPING GONG \& HEZHI XIE
}

\begin{abstract}
Excessive exercise leads to myocardial injury or even sudden exercise death. For the vast sports population, appropriate physiological state is a necessary condition for exercise. The present study aims to investigate the cardioprotective effects and potent mechanism of astragalus polysaccharide (APS) treatment against the exerciseinduced myocardial injury via in vitro cell-based assay and in vivo model rat. Efficacies of APS incubation on the inflammatory response and oxidative stress induced by LPS were both explored in $\mathrm{H} 9 \mathrm{C} 2$ cells by using CCK-8 and western blotting method, respectively. Normal SD rats were randomly divided into saline-treated overexercise rat group, and APS-treated overexercise rat groups with three doses. Then long-term swimming training load cycle (8 week) were performed on these rats. Finally, the changes on body weight, myocardial morphological and injury indicators, as well as the inflammation-related proteins in overexercise-induced model rats were all assessed. Three concentrations of APS all significantly increased cell viability, and decreased the apoptosis of cardiomyocytes in LPS-treated H9c2 cells. Moreover, chronic treatment of APS at all three doses also could obviously decreased myocardial injury-related indicators. Furthermore, the histopathologic examination exhibited that the APS successfully attenuated the changes of myocardial tissues, reduced the lipid accumulation and the protein levels of $I L-1 \beta$, TNF- $\alpha$ and NF-KB. Furthermore, the APS could activate the AMPK signaling pathway, enhance the autophagy and suppress the production of ROS. On conclusions, APS exerted the protective efficacies on overexercise-induced myocardial injury by activating the AMPK signaling pathway to increase autophagy and suppress the inflammation response, oxidative stress, apoptosis of myocardial cells.
\end{abstract}

Key words: Astragalus polysaccharide, Myocardial injury, Excessive exercise, Autophagy, Inflammation, AMPK signaling pathway.

\section{INTRODUCTION}

In recent years, sudden deaths during sports have occurred from time to time. Scientific research has found that exercise training can produce adaptive changes in the morphological structure and physiological function of the human heart (Fletcher et al. 2013). In untrained subjects, the increase in cardiac output mainly relies on an increase in heart rate, while professional athletes increase the output per beat. In this case, compensatory cardiomegaly occurs during long-term aerobic exercise, and cardiac load also increases during exercise (Fletcher et al. 2013). With the further deepening of exercise-heart research, scientists have found that sudden exercise death is associated with cardiac and cerebrovascular diseases, and other factors also include heat stroke, failure caused 
by excessive physical consumption, and drug abuse. Sudden cardiac death is the common cause of sudden exercise death, accounting for about $80 \%$. Hypertrophic cardiomyopathy is the leading cause of sudden cardiac death in young athletes, and cardiac morphology can be altered through long-term strenuous exercise. A European survey showed that 2354 Olympic athletes with a mean age of only 27.6 years had a heart disease rate as high as $7.3 \%$. Of course, ordinary fitness people are also sudden sports death people, especially when they are already too tired (Kaikkonen et al. 2010, Kwak 2013). However, in order to improve the athletic ability and competitive level of athletes, competitive sports often stimulate the body with long-term and large-load training (Parker et al. 2014, Schultz et al. 2007). Affected by large load, long training cycle, short recovery time and other factors, it often causes overtraining syndrome in the body and induces pathological conditions of multiple organ dysfunction (Ghorayeb 1995, Liu 2013). Studies have shown that overtraining can evoke not only exercise-induced cardiac overload, but also the damage of local tissue structure of the myocardium, and trigger a cascade of pathological and functional alterations (da Rocha et al. 2018, Ghorayeb 1995).

Currently, how to alleviate exercise-induced myocardial injuryis a problem demanding prompt solution of sports medicine (Zhang et al. 2019a). Both domestic and foreign studies have confirmed that some naturally occurring compounds, such as astaxanthin, have antioxidant activity and can improve the body's immunity and prevent the occurrence and development of cardiovascular diseases, including myocardial injury, and other diseases (Gai et al. 2020, Xie et al. 2020).

Astragalus membranaceus has the pharmacological effects, including strengthening vital energy, tonifying qi and other protective efficacies, which could reduce the adverse reactions of chemotherapeutic drugs, improve the immune function of the body, and thus improve the life quality of patients (Auyeung et al. 2016, Fu et al. 2014). The main component of Astragalus membranaceus, Astragalus polysaccharide (APS), has many pharmacological effects, including antihyperglycemic, antihypertensive, anti-viral, antioxidative and immune-enhancing activities (Li et al. 2020, Zhang et al. 2019b). Previously research found that APS can down-regulate the expression of NF-KB pathway, thereby inhibiting myocardial apoptosis produced after cardiac hemorrhage in rats, suggesting that APS can improve the damage of myocardial tissue and has the potential to be a candidate molecule for improving myocardial injury, which is worthy of in-depth study (Liu et al. 2019, Xu et al. 2020).

In this study, APS was used to investigate the ameliorative effects after nutritional intervention in an overtrained rat model with myocardial injury. The protective effect of APS on exercise-induced myocardial injury in rats and its potential mechanism were finally judged by testing the serum and tissue levels of myocardial injury markers, oxidative stress and inflammation-related factors, as well as observing the damaged myocardial tissues in rats via HE staining and immunohistochemistry methods, in order to provide a theoretical basis for the clinical application of APS in the field of sports nutrition and sports medicine.

\section{MATERIALS AND METHODS}

\section{Materials}

Astragalus polysaccharide with purity over 85\% were obtained from Tibobiochemistry Company (Beijing, China). The H9C2 cells, a kind of rat heartderived cells, were obtained from ATCC (Manassas, USA). The Dulbecco's modified Eagle's medium (DMEM), penicillin, 
streptomycinandfetal bovine serum were purchased from Thermo Fisher (USA). Thetrypsin, LPS, and CCK-8 kits were all brought from Beijing Solarbio Company (Beijing, China). The cellular apoptosis and ROS measurement kits were brought from Shanghai YoungChan Company (Shanghai, China). All antibodies were all brought from Abcam company (Cambridge, UK).

\section{Cell culture and detection}

The H9C2 cell was incubated in the DMEM containing the $1 \%$ penicillin and streptomycin and $10 \%$ FBS in thermostatic incubator at $37^{\circ} \mathrm{C}\left(5 \% \mathrm{CO}_{2}\right)$. After cell recovery is complete, the cells when grown to $75 \%$ were digested by using the trypsin and then seeded into 12 well plate for further culture. In addition, the H9C2 cell wasassigned into the normal control group without the treatment of LPS and APS, LPS (1 $\mu \mathrm{g} / \mathrm{mL})$ group, LPS $(1 \mu \mathrm{g} / \mathrm{mL})+$ APS $(10 \mu \mathrm{g} / \mathrm{mL})$ group, LPS $(1 \mu \mathrm{g} / \mathrm{mL})+$ APS $(30 \mu \mathrm{g} / \mathrm{mL})$ group, LPS $(1 \mu \mathrm{g} / \mathrm{mL})+$ APS $(90 \mu \mathrm{g} / \mathrm{mL})$ group and then treated for 24 hours. The cellar viability of $\mathrm{H} 9 \mathrm{C} 2$ was detected in accordance with the protocol of CCK-8 kit. Cell viability was calculated with the following formula. Moreover, the cellular ROS levels in $\mathrm{H} 9 \mathrm{C} 2$ cells were measured by flow cytometry method.

Cell viability $(\%)=\frac{\text { OD450 in experimental group-OD450 in blank group }}{\text { OD450 in control group-OD450 in blank group }} \times 100 \%$

\section{Animal grouping and training experiment method}

After 4 days of adaptive feeding, the rats were screened with $20 \mathrm{~min} / \mathrm{d}$ exercise for 3 days, individual maladapted swimmers were eliminated, and rats were excluded from the experiment if they did not adhere to quality requirements. The remaining rats were divided into 4 groups by digital randomization: quiet control group ( $n=12)$, overtraining group $(n=12)$, and three doses of APS+ overtraining group
(10, 30 and $90 \mathrm{mg} / \mathrm{kg}, \mathrm{n}=12$ ). The professional gavage device was used for gavage once a day, the gavage volume of APS was $5 \mathrm{~mL} / \mathrm{kg}$, and the other groups were also gavaged with the same amount of saline. All the experimental animal experiments were applied according to the standard procedures compiled by the animal experiment center of SLbio technology Co., Ltd. (China) with approval No. CDI-201809-015.

The quiet control group was routinely housed without exercise and without any intervention. The general training group underwent formal moderate-intensity swimming training for 8 weeks (non-weight-bearing), 6 days a week, once a day. The first swimming train was performed for $20 \mathrm{~min}$, and then gradually increased, by 60 min per day at the end of the first week, to 90 min per day at the end of the second week, to 120 min per day at the end of the third week, and this amount of exercise was maintained for the following 5 weeks. For the first 3 weeks of training time arrangement, the overtraining group and the astaxanthin intervention group were the same as the general training group, but the rats had a negative $0.5 \%$ body weight during training. High-intensity training was scheduled from the fourth week, with each training to exhaustion. Exhaustion criteria were based on the fact that rats did not show water $10 \mathrm{~s}$ after subsidence. $1 \%$ body weight at week 4 and 2\% body weight at week 5 , training once a day. The 6 th week was $2 \%$ body weight, and training was performed twice a day (morning/afternoon). In weeks 7-8, training was performed thrice a day (morning/ afternoon/night), all of which were negative for $5 \%$ body weight.

Immediately after swimming training, $2 \%$ pentobarbital sodium was used for deep anesthesia, and $5 \mathrm{~mL}$ was taken from the abdominal artery. Sodium citrate anticoagulation solution was added, and after $30 \mathrm{~min}$ in a circulating water bath at $37{ }^{\circ} \mathrm{C}$, serum was 
separated and prepared by centrifugation at $4000 \mathrm{rpm}$ for $10 \mathrm{~min}$. After blood collection, the thoracic cavity was immediately cut open, the heart was removed, and about $0.5 \mathrm{~g}$ of myocardial tissue from the anterior wall of the left ventricle was taken to make a $10 \%$ tissue homogenate. The supernatant which obtained from homogenate after centrifugation at 4000 rpm for 30 min was taken for measurement. Myocardial MDA content and SOD activity were measured by xanthine oxidase method and TBA method, respectively. Serum and myocardial total ET and CGRP levels were measured by radioimmunoassay. CTnI, SOD, MDA, ET and CGRP were detected by using ELISA kits in strict accordance with the instructions (Nanjing Jian Cheng Bioengineering Company). After the heart were removed, washed, and frozen, they were cut into four transverse slices parallel to the atrioventricular ring, then fixed in $4 \%$ buffered formalin and stained by hematoxylin-eosin $(H \& E)$.

\section{Western blotting measurement}

The 15\% SDS-PAGE was applied to separate the cardiac tissue proteins. The separated proteins were electro-transferred to PVDF membrane and further blocked by using the 5\% BSA for 2 hours at $25{ }^{\circ} \mathrm{C}$, followed by incubating the primary antibody against different indicators overnight at $4{ }^{\circ} \mathrm{C}$. In addition, the PVDF membrane were developed using enhanced chemiluminescence after being incubated with HRP-labeled secondary antibody at the dilution of 1:4000. Furthermore, the PVDF membrane was further scanned and calculated via the Typhoon and Image J, respectively.

\section{Data statistics}

Data statistics were performed using SPSS 13.0 software for data processing, and the data were expressed as $\pm \mathrm{S}$. Analysis of variance was used for comparison between multiple groups, and only $P$ value less than 0.05 were considered statistically significant.

\section{RESULTS}

\section{APS improved the LPS-induced cell viability and apoptosis of $\mathrm{H} 9 \mathrm{C} 2$ cells}

The H9C2 cellular viability in each group were evaluated via the CCK-8 assay. As the result showed in Figure 1, co-incubation with LPS obviously reduced the survival rate of $\mathrm{H} 9 \mathrm{C2}$ cells compared to the normal control group. In contrast, the cellular viabilities of all three LPS+APS $(10,30$ and $90 \mu \mathrm{g} / \mathrm{mL})$ treated H9C2 cell groups were obviously improved which are all distinctly higher than that of the LPS alonetreated cells (all $p<0.05$ ) in a significant dose dependent model. In addition, flow cytometry method was used to quantify the distribution of late apoptotic cells. Compared with the control group, apoptotic rate in the LPS treated only H9C2 group was significantly up-regulated. In contrast, late apoptosis in all three LPS+APS (10, 30 and $90 \mu \mathrm{g} / \mathrm{mL})$ co-incubation group were significantly improved than that in LPS only group, and all the results showed statistically significant differences $(p<0.01)$.

Western blot analysis also showed the similar results in the expression of apoptosis associated proteins. As shown in Figure 2, LPS challenge significantly upregulated the protein expression levels of Bax and Caspase 3, while down-regulated the expression of BCL2 (all $p$ $<0.01)$. In addition, three concentration of APS treatment $(10,30$ and $90 \mu \mathrm{g} / \mathrm{mL}$ ) all markedly decreased and increased expression of Bax and BCL2 protein, respectively (both $p<0.05$ ). Moreover, the protein level of Caspase 3 was obviously decreased in both middle and high doses of APS (30 and $90 \mu \mathrm{g} / \mathrm{mL}$ ) treated groups compare to the LPS-treated group $(p<0.05)$. 
$\mathbf{a}$

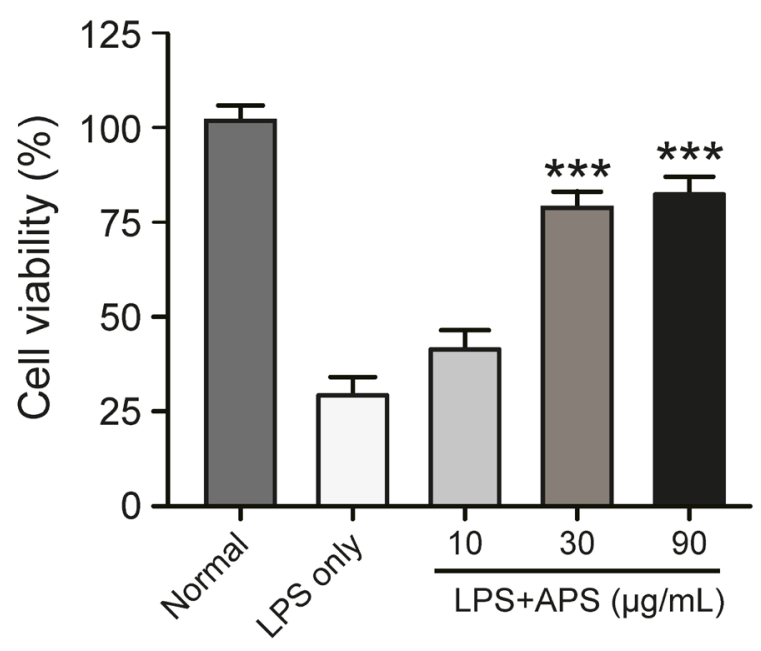

b

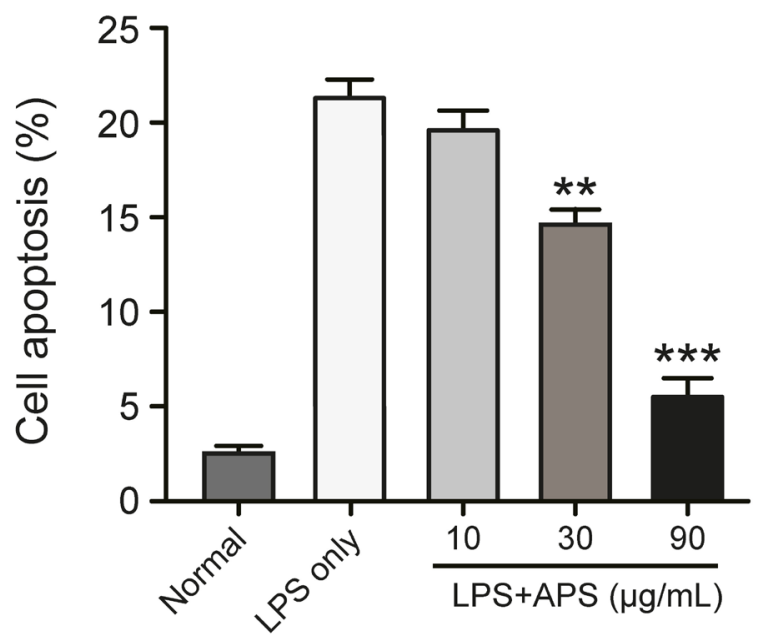

Figure 1. Effects of APS incubation on (a) cellular viability and (b) cellular apoptosis of H9C2 cell. ${ }^{*}=p<0.05$, ${ }^{* *}=p<0.02,{ }^{* * *}=p<0.001$ vs. LPS treated only cell group. Values represent mean \pm SD of 5 independent experiments.

Western blot analysis also showed the similar results in the expression of apoptosis associated proteins. As shown in Figure 2, LPS challenge significantly upregulated the protein expression levels of Bax and Caspase 3, while down-regulated the expression of BCL2 (all $p<0.01)$. In addition, three concentration of APS treatment $(10,30$ and $90 \mu \mathrm{g} / \mathrm{mL}$ ) all markedly decreased and increased expression of Bax and BCL2 protein, respectively (both $p<0.05$ ). Moreover, the protein level of Caspase 3 wa sobviously decreased in both middle and high doses of APS (30 and $90 \mu \mathrm{g} / \mathrm{mL}$ ) treated groups compare to the LPS-treated group $(p<0.05)$.

\section{APS ameliorated the myocardial injury in overtraining rats}

As illustrated in Figure 3, the serum levels of $\mathrm{LDH}$ and $\mathrm{CK}$ in saline-treated excessive exercise group were significantly higher than that of the normal rat group suggesting occurrence of myocardial injuries after long-term overtraining. Nevertheless, the serum levels of LDH and CK in three APS treated groups were all obviously reduced compared to those in saline treated control group and the improvement were observed in a dose-dependent manner. Compared with the normal control group, the serum content of cTnI in the saline-treated overtraining group was significantly increased, and the overtraining ones treated with three doses of APS were also significantly increased (all $p<0.05$ ), but significantly lower than that in the overtraining control group $(10 \mathrm{mg} / \mathrm{kg}$, $p<0.05 ; 30 \mathrm{mg} / \mathrm{kg}, p<0.01 ; 90 \mathrm{mg} / \mathrm{kg}, p<0.001)$, and the improvement effects appeared to be dose dependent.

Additionally, overexercise rats had obviously higher serum MDA content than normal ones, suggesting the progressive oxidative stress in cardiac tissues after the chronic excessive exercise. In contrast to this, the APS treated rats at 10, 30 and $90 \mathrm{mg} / \mathrm{kg}$ had significantly down-regulated serum MDA levels than salinetreated excessive exercise rat group (all $p<0.05$ ), suggesting that chronic treatment of APS can 
A

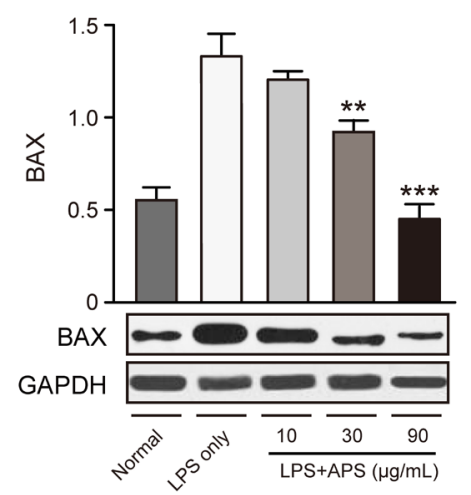

B

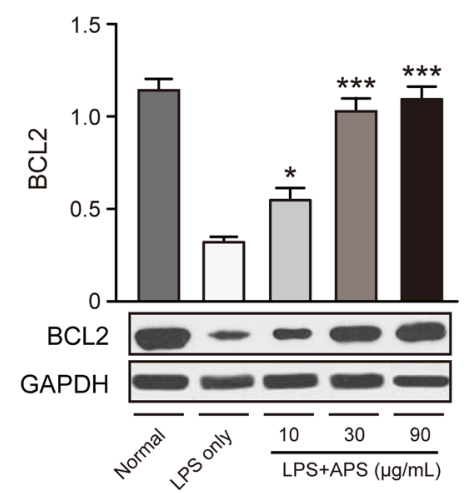

C

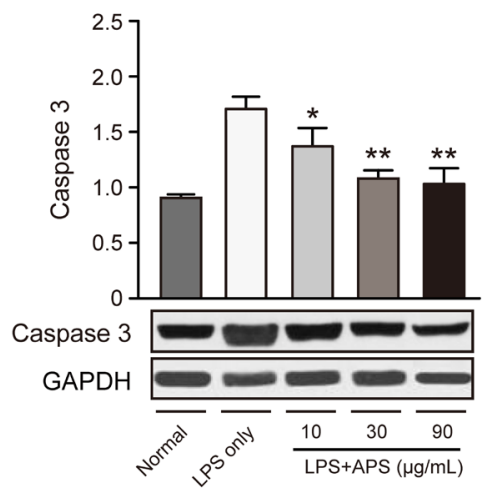

Figure 2. Effects of APS incubation on the expression of the apoptosis-related indicators. The expression analysis of (a) BAX, (b) BCL2 and (c) Caspase 3 in western blotting detection. ${ }^{*}=p<0.05,{ }^{* *}=p<0.02,{ }^{* * *}=p<0.001$ vs. LPS treated only cell group. Values represent mean \pm SD of 5 independent experiments.

reduce the oxidative stress reaction as well as the free radicals induced damage on myocardial tissues of model rats. Likewise, the differences of T-SOD activities of different model rat group also indicated that chronic treatment of APS held promising anti-oxidant efficacies, which obviously upregulated the serum level of SOD and finally improve the damage of oxygen free radical to the cardiac tissues.

Furthermore, as shown in Table I, the contents of ET in serum and myocardial tissue were significantly increased in hyper-exercised rats when compared with the normal rats (all $p<0.05)$. Rats in the overtraining group that were chronically administered APS were notably decreased when compared with the salinetreated overtraining group at all three doses (all $p<0.01$ ). In addition, the contents of CGRP in serum and myocardium were significantly reduced in the overtraining group when compared with the normal control group, and the difference was most obviously in salinetreated overtraining rats. Furthermore, these changes were significantly reversed in APS treated overtraining groups at all three doses (all $p<0.01$ ).

\section{Effects of chronic treatment of APS on the myocardial morphological changes of the exercise rat model}

The pathological change of myocardial tissue in overtraining rats which received longterm excessive-exercise and cardioprotective efficacies of APS at three dosages were evaluated by $H \&$ Estaining test. As the results shown in Figure 4 , the myocardial structure of the normal rat was completed with clear boundary, and cardiac cells arranged regularly without significant lesion and infiltration of inflammatory cells. However, the section in saline-treated excessive-exerciser at group was completely disordered with the blurred myocardial cell edge, shrunken nucleus, vacuole-like lesion and some seep inflammatory cells. Significantly, the pathological results of the cardiac tissue from APS treated model rats were improved without above deterioration features, indicating the potent cardioprotective efficacies of APS on myocardial injury.

\section{Effect of long-term APS administration on inflammation related protein expression levels in the myocardial tissues of model rats}

The inflammation related indicators expression levels, including IL-1 $\beta$, TNF- $\alpha$ and NF-KB in 
a

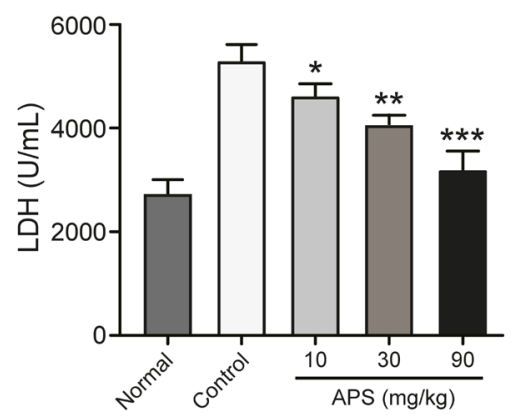

b

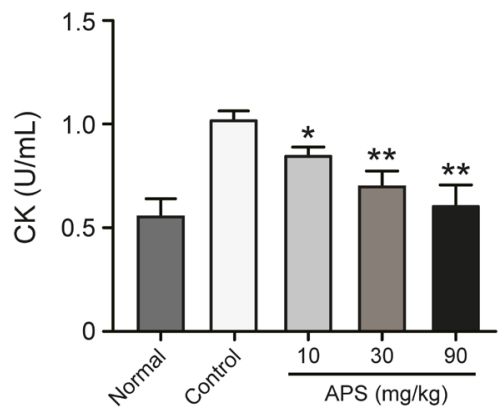

C

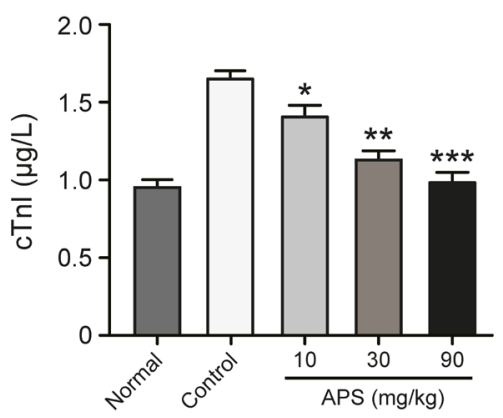

d

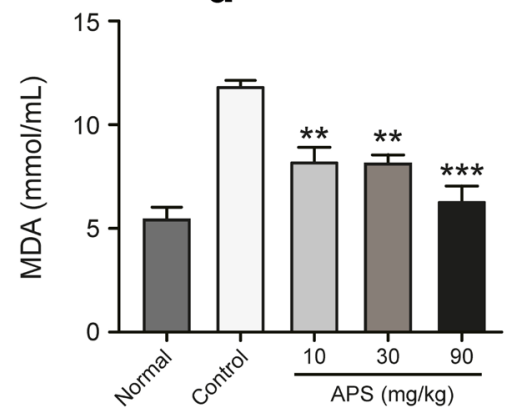

$\mathbf{e}$

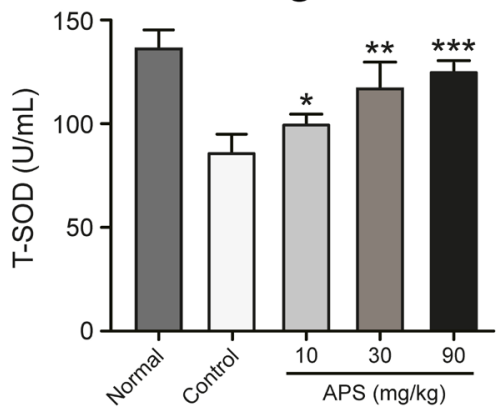

Figure 3. Efficacies of APS treatment on serum cardiac markers in overtraining rats. The concentration of (a) LDH, (b) CK, (c) CTnl, (d) MDA and (e) T-SOD in rat serum. ${ }^{*}=p<0.05,{ }^{* *}=p<0.02,{ }^{* * *}=p<0.001$ vs. saline-treated excessive exercise rat group. Values represent mean \pm SD of 8 independent experiments.

cardiac tissues were detected via utilizing the western blot method. As the results shown in Figure 5, chronic treatment of APS at dose of 10, 30 and $90 \mathrm{mg} / \mathrm{kg}$ all significantly reduced the expression levels of these three proteins indicating the occurrent inflammatory response in the myocardium. Moreover, the expression levels these three inflammatory factors were all obviously down-regulated after the chronic 0.9 $\mathrm{mg} / \mathrm{kg}$ APS administration $(p<0.001)$ revealing that the APS could alleviate the inflammatory response thus protect the heart from the overtraining induced inflammatory injury.

\section{APS improved autophagy and inhibited the production of ROS in excessive exercise model rats via activating the AMPK signaling pathway}

As the results shown in Figure 6, the protein expression of AMPK including AMPK phosphorylation (p-AMPK) and total AMPK (t-AMPK), as well as autophagy related markers including Parkin and Beclin1, in myocardial tissues of model rats were measured using the WB method. Compared to the saline alone treated model rats, the expression levels of $\mathrm{p}$-AMPK in all three APS groups (10, 30 and $90 \mathrm{mg} / \mathrm{kg}$ ) were all obviously upregulated (all $p<0.05$ ). The t-AMPK level also expressed at a similar trend APS treated groups (all $p<0.05$ ). In addition, we further investigated whether the chronic treatment of APS can reverse the decrease of the autophagy-related proteins in excessive exercise model rats. As the results showed in the Figure $6 c-d$, the autophagy markers Beclin1 and Parkin were all significantly reversed by three concentration of APS treatment $(p<0.05)$. Furthermore, ROS expression in different model rat groups were furtherly assessed via flow 
Table I. The contents of ET and CGRP in the rat serum and myocardium.

\begin{tabular}{|c|c|c|c|c|c|}
\hline \multirow{2}{*}{ Group } & \multirow{2}{*}{ Treatment } & \multicolumn{2}{|c|}{ ET } & \multicolumn{2}{c|}{ CGRP } \\
\cline { 2 - 6 } & & Serum $(\mathbf{p g} / \mathbf{m L})$ & Tissue $(\mathbf{p g} / \mathbf{g})$ & Serum $(\mathbf{p g} / \mathbf{m L})$ & Tissue $(\mathbf{p g} / \mathbf{g})$ \\
\hline \multirow{2}{*}{ Normal rats } & Saline & $179.2 \pm 23.4$ & $17.3 \pm 2.5$ & $97.3 \pm 12.8$ & $247.3 \pm 52.1$ \\
\hline \multirow{2}{*}{$\begin{array}{c}\text { Overtraining } \\
\text { rats }\end{array}$} & Saline & $286.3 \pm 17.2$ & $38.4 \pm 8.5$ & $21.4 \pm 1.8$ & $120.2 \pm 15.2$ \\
\cline { 2 - 6 } & $10 \mathrm{mg} / \mathrm{kg}$ APS & $251.7 \pm 27.6^{*}$ & $25.2 \pm 6.9^{* *}$ & $45.2 \pm 10.1^{* *}$ & $161.2 \pm 21.8^{*}$ \\
\cline { 2 - 6 } & $30 \mathrm{mg} / \mathrm{kg}$ APS & $219.8 \pm 56.8^{* *}$ & $24.4 \pm 5.3^{* *}$ & $54.6 \pm 14.2^{* * *}$ & $164.1 \pm 13.4^{*}$ \\
\cline { 2 - 6 } & $90 \mathrm{mg} / \mathrm{kg}$ APS & $197.2 \pm 19.5^{* * *}$ & $21.7 \pm 7.5^{* *}$ & $72.5 \pm 17.2^{* * *}$ & $212.1 \pm 31.4^{* * *}$ \\
\hline
\end{tabular}

${ }^{*}=p<0.05,{ }^{* *}=p<0.02,{ }^{* * *}=p<0.001$ vs. saline-treated excessive exercise rat group.

Normal

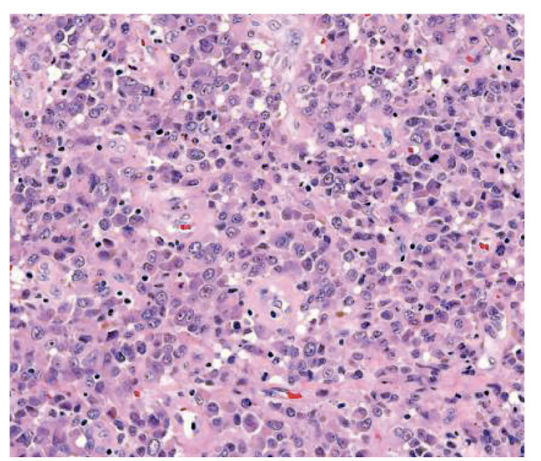

Control

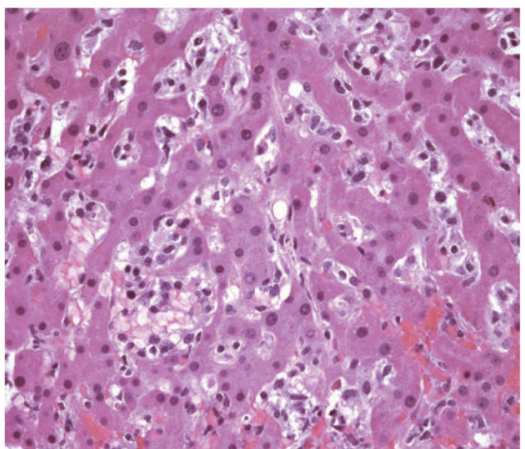

APS (10 mg/kg)

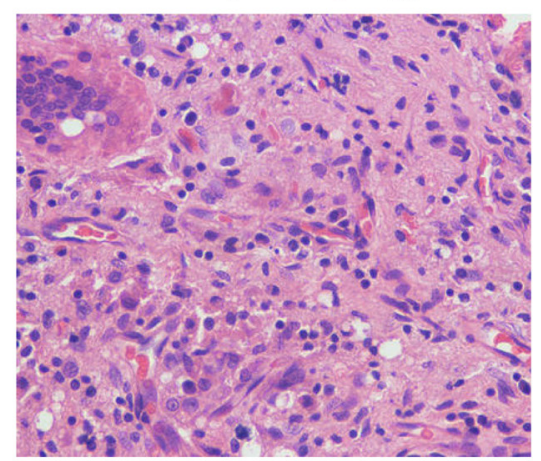

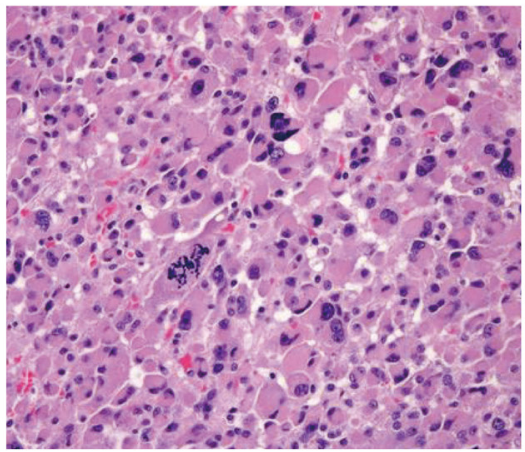

APS (30 mg/kg)

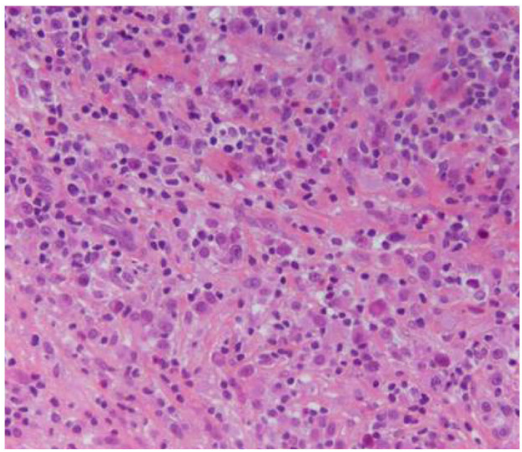

APS $(90 \mathrm{mg} / \mathrm{kg})$

Figure 4. Cardioprotective efficacies of chronic treatment of APS at different doses on the cardiac injuries in excessive exercises-induced model rats.

cytometry method (data not shown) and the results showed in Figure 6e proved that all the three doses of APS obviously down-regulated the ROS production $(p<0.01)$.

\section{DISCUSSION}

Exercise is a double-edged sword, excessive exercise will lead to myocardial injury or even sudden exercise death, too little exercise will bring other health problems. For the majority of student groups, appropriate physical exercise 
$\mathbf{a}$

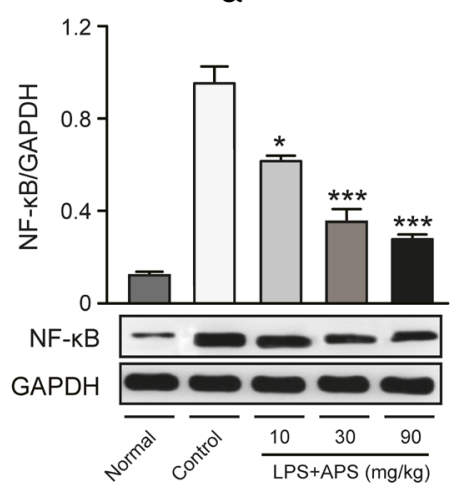

b

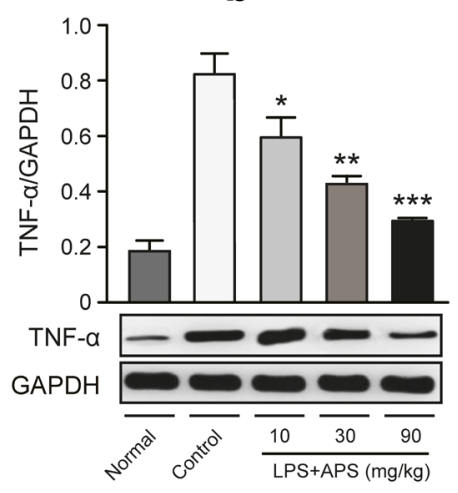

C

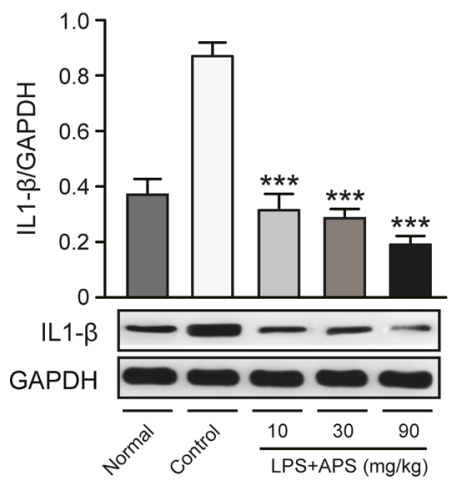

Figure 5. Chronic treatment of APS prevented the overtraining induced myocardial inflammation. The protein expression levels of (a) NF-KB, (b) TNF- $\alpha$ and (c) IL-1 $\beta$ were detected via WB method. ${ }^{*}=p<0.05,{ }^{* *}=p<0.02$, $* * *=p<0.001$ vs. saline-treated excessive exercise rat group. Values represent mean \pm SD of 8 independent experiments.

or advantages outweigh disadvantages, but to avoid excessive fatigue. For example, students in order to physical examination or physical examination, heavy exercise, may lead to sudden cardiac overload, causing sudden death. Sporthealth portable monitoring devices are not currently able to monitor the overall condition of an athlete's heart and body function at the molecular level. With the development of sports science, how to carry out physical exercise and scientific training is well documented, but the problem of excessive exercise or excessive fatigue cannot be treated from the perspective of exercise alone, and should be judged from a wider perspective, such as dietary nutrition, routines, body sensation, etc., from the perspective of recent times, in addition to learning and mastering more knowledge about myocardial injury from the perspective of sports medicine, which is more conducive to the judgment of their own feelings.

Cardiomyocytes are mainly metabolized aerobically with extremely strong oxygen uptake capacity (Suresh et al. 2016). Previous studies have shown that the myocardium mainly relies on the dilatation of coronary vessels to increase blood flow during exercise, thereby meeting the oxygen demand of the myocardium (Duncker \& Bache 2008). During strenuous exercise, coronary blood flow can even reach four times that of quiet (Duncker \& Bache 2008). The myocardium is extremely vulnerable to hypoxia flow when the reduction of coronary blood flow happened due to the damage of coronary vascular structure or function (Duncker \& Bache 2008). Appropriate exercise load can promote adjustable and physiological remodeling of cardiac structure and help improve cardiac function (Bove 2016). However, acute exhaustion and high-intensity physical exercise will have adverse effects on the body such as oxidative stress, and even cause exercise-induced myocardial injury (da Rocha et al. 2018, O’Keefe et al. 2012).

Astragalus membranaceus is one of the commonly used traditional Chinese medicine characterized by sweet taste, warm nature and the numerous benefit effects (Fu et al. 2014). At present, Astragalus injection has been widely used in clinical practice and plays a significant role in treating cardiac diseases (Ma et al. 2013, Su et al. 2009). Many previous studies have shown that Astragalus membranaceus injection 
a

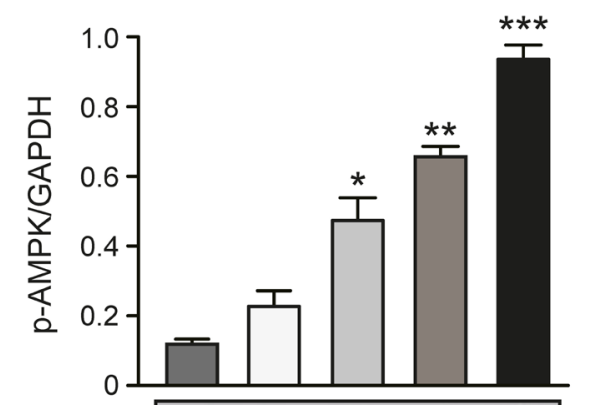

p-AMPK

GAPDH

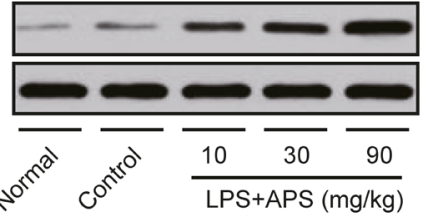

C

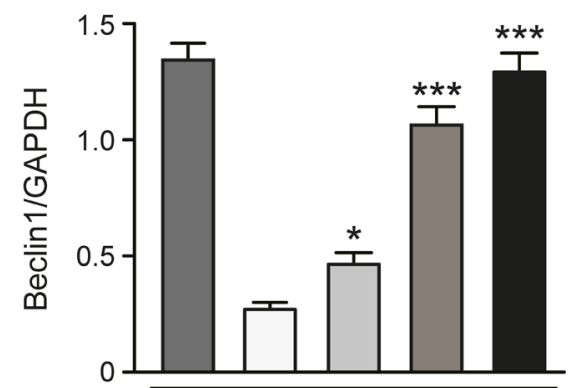

Beclin1

GAPDH

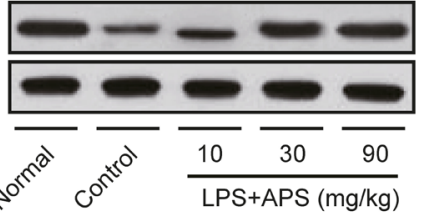

b

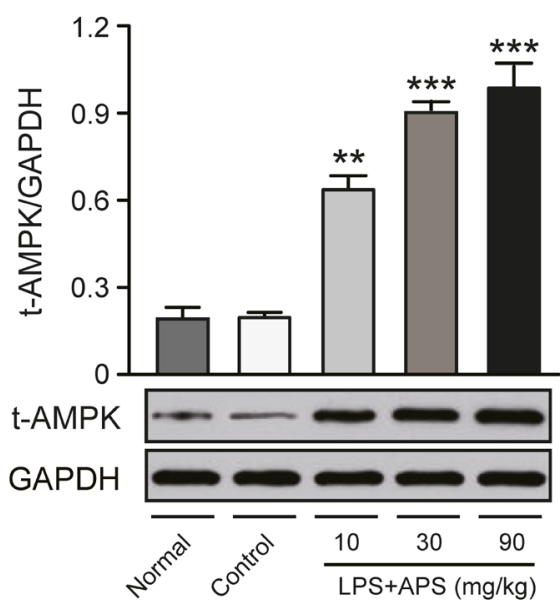

d

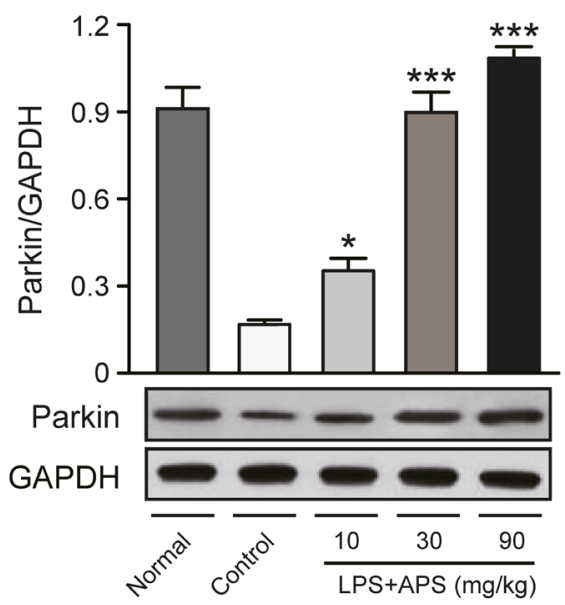

Figure 6. Chronic efficacies of APS treatment on AMPKdependent autophagic signaling in model rats. The protein relative expression of (a) p-AMPK/ GAPDH, (b) t-AMPK/ GAPDH, (c) Beclin 1/ GAPDH, (d) Parkin/ GAPDH and (e) ROS content. ${ }^{*}=p<0.05$, $* *=p<0.02$, $* * *=p<0.001$ vs. saline-treated excessive exercise rat group. Values represent mean \pm SD of 6 independent experiments.

e

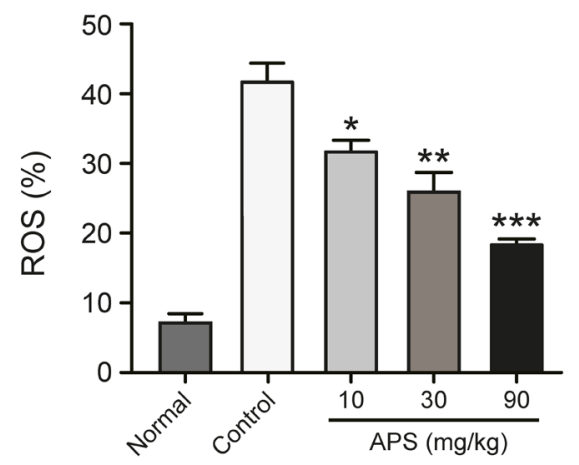


can inhibit the development of left ventricular hypertrophy and prevent myocardial fibrosis (Wei et al. 2020). Astragalus polysaccharide (APS), a polysaccharide extracted from Astragalus membranaceus, is one of the main active components of Astragalus membranaceus and has a wide range of pharmacological effects in the treatment of heart diseases and immune diseases (Li et al. 2020). At the same time, APS have also been reported to have important roles in anti-hyperglycemia, anti-tumor, and anti-oxidation (Li et al. 2020, Zhang et al. 2019b). Studies have confirmed that APS can improve the cell survival rate of injured myocardium caused by hypoxia/reoxygenation process, and reduce the apoptosis rate of cardiomyocytes (Zhou et al. 2018). Previous experiments confirmed that APS can inhibit isoproterenol-induced myocardial hypertrophy and protect the myocardium in rats by regulating PGC-1a (Dai et al. 2014). Then, it is unclear whether the protective effect of APS can be through inhibiting cardiomyocyte damage or even apoptosis resulting from excessive exercise.

In this study, in vitro experiments showed that the LPS induction significantly increased H9C2 cells apoptosis accompanied by significant improvement of the apoptosis-related proteins expression suggesting the protected potential of APS treatment in reducing apoptosis. CGRP is the strongest vasodilator known so far in the human body, which can significantly relax coronary vessels, reduce blood viscosity and vascular resistance of coronary vascular, increase cardiac blood flow and oxygen delivery, and play a major role in regulating of local blood flow in the heart (Tippins 1986). CGRP and endothelin (ET) are two vasoactive substances that are completely opposite in their regulatory effects on blood pressure and blood circulation (Hartopo et al. 2020, Tippins 1986). The two have a strong and long-lasting mutual antagonistic effect on blood vessels. Under normal physiological conditions, the secretion of the two substances is in a dynamic balance, excessive release of endothelin will inhibit the secretion of calcitonin gene-related peptide, and the imbalance of their secretion will lead to the occurrence of diseases. As the "gold standard" for the diagnosis of myocardial injury, cTnl is highly specific.

The increase of free radicals caused by prolonged overtraining can trigger morphological changes and abnormal function of endothelial cells (EC) through the oxidative modification of LDL and HDL. Changes in EC morphology and abnormal function lead to excessive secretion of end othelial cells, which in turn triggers increased ET levels in plasma and myocardial tissue. It has also been shown that myocardial ischemia, hypoxia, and injury during prolonged strenuous exercise, can also lead to increased ET levels in plasma and myocardial tissue. However, CGRP, which antagonizes ET, is continuously consumed due to the increase of ET, and the vasoconstrictor effect of ET cannot be completely antagonized, causing cardiovascular contraction to decrease cardiac supply, further strengthen myocardial ischemia and hypoxia injury, and also further inhibit CGRP secretion in the myocardium, which in turn leads to a decrease in CGRP levels in plasma and myocardial tissue.

In these experiments, the serum cTnI content in saline-treated overtrained rats was significantly increased compared with the normal group ( $p<0.05)$, indicating that 8 -week swimming training with incremental load has led to overtraining syndrome in rats due to factors such as long duration cycle, high intensity, and short recovery time, which subsequently triggers exercise-induced myocardial injury in rats.

Myocardial tissue is poorly protected against oxygen free radicals but highly sensitive to peroxidation. The excessive free radicals produced by overtraining can result 
in exercise-induced myocardial injury, and the extent of injury is closely associated with the level of free radicals and lipid peroxidation. Antioxidant enzymes are the main substances that scavenge free radicals in the body. SOD, as the only enzyme with oxygen radicals as substrates, is highly specific for substrates and has extremely strong catalytic potency. It can scavenge superoxide anions by catalyzing the formation of hydrogen peroxide from superoxide anions and plays an important role in preventing and reducing free radical damage and mitochondrial damage in myocardial tissue. At the same time, SOD can also act synergistically with GSH-Px to catalyze the reduction reaction of GSH to hydrogen peroxide, thus playing a role in protecting the structural and functional integrity of cell membranes. MDA, as the main product of cellular lipid peroxidation, is now recognized as a sensitive indicator of free radical metabolism in the body. ET is a vasoactive peptide with multiple biological effects, widely distributed in the body, and is currently known as the endogenous vasoconstrictor with the strongest vasoconstrictor effect and the longest duration of action in vivo, which can exert vasoconstrictor effects by binding to endothelin receptors. It helps to stabilize cardiovascular function at physiological concentrations and plays a major role in regulating systemic vascular and local blood flow. Oxidative stress response has been considered to be one of the most key factors in hyperexercise-induced myocardial injury pathogenesis.

In this study, serum cTnl content, serum and myocardial ET, and myocardial MDA content were significantly lower in the APS + overtraining group than that in overtraining group (all $p<$ 0.05). The contents of SOD in myocardium, CGRP in serum and myocardium were significantly increased (all $p<0.01$ ). These results suggest that APS supplementation can not only increase the in vivo activities of SOD and GSH-px in the body, but also decrease the occurrence of lipid peroxidation and the generation of free radicals, and then maintain the dynamic balance of antioxidant defense system in the body, which plays a positive role in scavenging excessive in vivo free radicals, alleviating the damage of free radicals to mitochondrial membrane and sarcoplasmic reticulum membrane, and alleviating the oxidative damage to enzyme proteins. Moreover, APS supplementation can effectively inhibit the increase of ET in plasma and myocardial tissue and ensure the relative balance of ET and CGRP concentrations by effectively increasing CGRP levels in plasma and myocardial tissue, dilating blood vessels in the appropriate range, improving myocardial blood supply, increasing myocardial oxygen supply, enhancing myocardial contractility, and increasing cardiac output, thus effectively preventing myocardial peroxidation and hyperexercise-induced injury.

It is very easy to understand that inflammatory factor release can promote the occurrence of inflammation and ultimately contributing to a continuous increase in ROS levels. Previous reports have shown that NF-kB, as a key factor in regulating signaling pathway closely related to systemic inflammatory reactions inflammatory, participated in the transcription and modulation of many inflammatory mediators. Reports have shown that the release of IL-1 $\beta$ and TNF- $\alpha$, which could be promoted by activated NF-KB activation, stimulated the production of inflammatory factors then result in excessive hypertrophy of cardiomyocytes, and finally irreversible damage to myocardial tissue fibrosis. In this study, we further found that long-term treatment of APS can significantly reduce the expression of all tested pro-inflammatory markers that 
lead to the notably reduction of inflammatory response in myocardial tissue.

AMPK, as an energy sensor, plays a key role in the regulation of cellular energy balance, but has been proposed as a key modulator of energy metabolism in heart. Phosphorylated AMPK involved in the cardiac energy metabolism in the body and can reduce cardiomyocyte injury. In the present study, in vivo experiments further showed that the expression levels of p-AMPK, Beclin1 and Parkin, three autophagyrelated proteins, were significantly increased in the heart tissue of overexercising model rats after APS treatment relative to the saline control group, suggesting that long-term administration of APS in the overexercising rat model can activate the AMPK signaling pathway, which in turn improves autophagy and cardiomyocyte apoptosis, in order to ultimately reduce longterm high-intensity exercise damage to the rat heart.

Neither instrumental testing nor treatment with drugs/health products can fundamentally change the root cause of excessive exercise occurrence. In sports life, the event of "sudden death by physical examination" frequently reflects the physical war of students, which should arouse the alarm and attention of education departments. We should intensify efforts to make physical education a compulsory course for higher education assessment, only in this way to urge many aspects to pay attention to physical education and students' physical health.

\section{REFERENCES}

AUYEUNG KK, HAN QB \& KO JK. 2016. Astragalus membranaceus: A Review of its Protection Against Inflammation and Gastrointestinal Cancers. Am J Chin Med 44: 1-22.

BOVE AA. 2016. Exercise and Heart Disease. Methodist Debakey Cardiovasc J 12: 74-75.
DAI H, JIA G, LIU X, LIU Z \& WANG H. 2014. Astragalus polysaccharide inhibits isoprenaline-induced cardiac hypertrophy via suppressing $\mathrm{Ca}^{2+}$-mediated calcineurin/ NFATc3 and CaMKII signaling cascades. Environ Toxicol Pharmacol 38: 263-271.

DA ROCHA AL ET AL. 2018. Excessive training induces molecular signs of pathologic cardiac hypertrophy. J Cell Physiol 233: 8850-8861.

DUNCKER DJ \& BACHE RJ. 2008. Regulation of coronary blood flow during exercise. Physiol Rev 88: 1009-1086.

FLETCHER GF ET AL. 2013. Exercise Standards for Testing and Training: A Scientific Statement From the American Heart Association. Circulation 128: 873-934.

FU J, WANG Z, HUANG L, ZHENG S, WANG D, CHEN S, ZHANG H \& YANG S. 2014. Review of the botanical characteristics, phytochemistry, and pharmacology of Astragalus membranaceus (Huangqi). Phytother Res 28: 1275-1283.

GAI YS, REN YH, GAO Y \& LIU HN. 2020. Astaxanthin protecting myocardial cells from hypoxia/reoxygenation injury by regulating miR-138/HIF-1 $\alpha$ axis. Eur Rev Med Pharmacol Sci 24: 7722-7731.

GHORAYEB N. 1995. The athlete heart. Physiological modifications $x$ overtraining and heart disease. Arquivos Brasileiros de Cardiologia 64: 161-165.

HARTOPO AB, SUKMASARI I, PUSPITAWATI I \& SETIANTO BY. 2020. Serum Endothelin-1 Correlates with Myocardial Injury and Independently Predicts Adverse Cardiac Events in Non-ST-Elevation Acute Myocardial Infarction. Int J Vasc Med 2020: 1-6.

KAIKKONEN P, RUSKO H \& MARTINMÄKI K. 2010. Post-exercise heart rate variability of endurance athletes after different high-intensity exercise interventions. Scand J Med Sci Sports 18: 511-519.

KWAK HB. 2013. Effects of aging and exercise training on apoptosis in the heart 9: 212-219.

LI W, HU X, WANG S, JIAO Z, SUN T, LIU T \& SONG K. 2020. Characterization and anti-tumor bioactivity of astragalus polysaccharides by immunomodulation. Int J Biol Macromol 145: 985-997.

LIU J. 2013. GW24-e3722 Ebselen protected myocardium from overtraining-induced oxidative damage in rats. Heart 99: A97.

LIU T ET AL. 2019. Astragalus polysaccharide from Astragalus Melittin ameliorates inflammation via suppressing the activation of TLR-4/NF-KB p65 signal pathway and protects mice from CVB3-induced virus myocarditis. Int J Biol Macromol 126: 179-186.

MA X, ZHANG K, LI H, HAN S, MA Z \& TU P. 2013. Extracts from Astragalus membranaceus limit myocardial cell death and improve cardiac function in a rat model of myocardial ischemia. J Ethnopharmacol 149: 720-728. 
O'KEEFE JH, PATIL HR, LAVIE CJ, MAGALSKI A, VOGEL RA \& MCCULLOUGH PA. 2012. Potential adverse cardiovascular effects from excessive endurance exercise. Mayo Clin Proc 87: 587-595.

PARKER L, MCGUCKIN TA \& LEICHT AS. 2014. Influence of exercise intensity on systemic oxidative stress and antioxidant capacity. Clin Physiol Funct Imaging 34: 377-383.

SCHULTZ RL, SWALLOW JG, WATERS RP, KUZMAN JA, REDETZKE RA, SAID S, DE ESCOBAR GM \& GERDES AM. 2007. Effects of Excessive Long-Term Exercise on Cardiac Function and Myocyte Remodeling in Hypertensive Heart Failure Rats. Hypertension 50: 410-416.

SU D, LI HY, YAN HR, LIU PF, ZHANG L \& CHENG JH. 2009. Astragalus Improved Cardiac Function of AdriamycinInjured Rat Hearts by Upregulation of SERCA2a Expression. Am J Chin Med 37: 519-529.

SURESH CB, NIRMAL P \& JASON RBD. 2016. The role of AMPK in cardiomyocyte health and survival. Biochimica et Biophysica Acta (BBA) - Molecular Basis of Disease 1862: 2199-2210.

TIPPINS JR. 1986. CGRP: a novel neuropeptide from the calcitonin gene is the most potent vasodilator known. J Hypertens Suppl 4: S102-105.

WEI Y, WU Y, FENG K, ZHAO Y, TAO R, XU H \& TANG Y. 2020. Astragaloside IV inhibits cardiac fibrosis via miR-135aTRPM7-TGF- $\beta$ /Smads pathway. J Ethnopharmacol 249: 112404.

XIE WJ, HOU G, WANG L, WANG SS \& XIONG XX. 2020. Astaxanthin suppresses lipopolysaccharide-induced myocardial injury by regulating MAPK and PI3K/AKT/mTOR/GSK3 $\beta$ signaling. Mol Med Rep 22: 3338-3346.

XU X, RUI S, CHEN C, ZHANG G, LI Z, WANG J, LUO Y, ZHU H \& MA $X .2020$. Protective effects of astragalus polysaccharide nanoparticles on septic cardiac dysfunction through inhibition of TLR4/NF-KB signaling pathway. Int J Biol Macromol 153: 977-985.

ZHANG H, LIU M, ZHANG Y \& LI X. 2019a. Trimetazidine Attenuates Exhaustive Exercise-Induced Myocardial Injury in Rats via Regulation of the Nrf2/NF-KB Signaling Pathway. Front Pharmacol 10: 175.

ZHANG Z, ZHANG L \& XU H. 2019b. Effect of Astragalus polysaccharide in treatment of diabetes mellitus: a narrative review. J Tradit Chin Med 39: 133-138.

ZHOU Q, MENG G, TENG F, SUN Q \& ZHANG Y. 2018. Effects of astragalus polysaccharide on apoptosis of myocardial microvascular endothelial cells in rats undergoing hypoxia/reoxygenation by mediation of the PI3K/Akt/ eNOS signaling pathway. J Cell Biochem 119: 806-816.

\section{How to cite}

TUO X, DENG Z, HUANG G, GONG H \& XIE H. 2021. Astragalus polysaccharide attenuates overexercise-induce myocardial injury via activating AMPK signaling pathway to suppress inflammation and oxidative stress. An Acad Bras Cienc 94: e20210314. DOI 10.1590/0001-3765202120210314.

Manuscript received on February 28, 2021;

accepted for publication on June 2, 2021

\section{XINLING TUO ${ }^{1}$}

https://orcid.org/0000-0002-0645-2193

\section{ZHIJIAN DENG ${ }^{2}$}

https://orcid.org/0000-0002-9376-9032

\section{GUOCHAO HUANG ${ }^{3}$}

https://orcid.org/0000-0002-4916-6349

\section{HUIPING GONG ${ }^{4}$}

https://orcid.org/0000-0002-9978-8090

\section{HEZHI XIE ${ }^{4}$}

https://orcid.org/0000-0003-0967-7336

${ }^{1}$ Guangdong Mechanical and Electrical Polytechnic, No.2 Chanchushi Rd. East, Tonghe, Baiyun

District, Guangzhou 510515, PR, China

${ }^{2}$ The Fifth Affiliated Hospital of Guangzhou Medical University, Harbour Road, n 621, Whampoa District, Guangzhou, Guangdong Province 510700, PR, China ${ }^{3}$ Guangzhou Liwan District Peizhen Primary School, n 4, Pingxi First Lane, Baihedong, Liwan District, Guangzhou, Guangdong Province 510380, PR, China ${ }^{4}$ Guangzhou Sport University, Guangzhou Road, n 1268, Guangzhou Avenue Middle, Tianhe District, Guangzhou, Guangdong Province 510500, PR, China

Correspondence to: Hezhi Xie and Huiping Gong

E-mail:thzxie@zjus.ac.cn,ghappy119@hotmail.com

\section{Author contributions}

XL Tuo: Conceptualization, Methodology, Data acquisition, Writing-original draft. ZJ Deng: Methodology, Data acquisition, Data analysis, Writing-review \& editing. GC Huang: Data acquisition, Data analysis, Validation, Writing-review \& editing. HZ Xie and HP Gong: Conceptualization, Supervision, Writingreview \& editing. All authors discussed the results and approved the final version of the manuscript.

\section{(cc) $\mathrm{BY}$}

